\section{Efeito moderador do risco social na relação entre risco biológico e desempenho funcional infantil}

\section{The moderating effect of social risk in the relationship between biologic risk and child functional performance}

\begin{abstract}
Objectives: to assess the impact of preterm birth and family socioeconomic status (SES) as they relate to functional and routine tasks performance in three years old children.

Methods: cross-sectional study including 40 children distributed into four groups according to biologic risk variables of high and low social risk. Children's abilities and independence were assessed by the standardized PEDI functional test in the areas of: selfcare, mobility and social function. Two-way ANOVA tested the interaction effect between biologic and social risks, and main effects of individual risk factors.

Results: significant interaction factors in mobility functional ability and independence in social function were demonstrated between preterm and at term children with low SES. Preterm children scored lower in the two aspects of functional performance. There were no significant differences in the groups of children with high SES in the evaluated areas.

Conclusions: social risk (moderating factor) changed the relationship between biologic and mobility abilities and social function independence. The interaction of biologic and social factors, and not only the presence of a sole risk factor impacts functional performance in three year old children. The results points towards increasing therapeutic actions favoring children exposed to biologic and social risk factors.
\end{abstract}

Key words Infant, premature, Child development, Socioeconomic factors, Risk factors, Biological factors
Marisa C. Mancini 1

Luiz Megale 2

Marina B. Brandão 3

Ana Paula P. Melo 4

Rosana F. Sampaio 5

1 Departamento de Terapia Ocupacional. Escola de Educação Física, Fisioterapia e Terapia Ocupacional. Universidade Federal de Minas Gerais. Unidade Administrativa II, $3 .^{\circ}$ andar. Campus Universitário. Pampulha. Av. Antônio Carlos, 6627. Belo Horizonte, MG, Brasil. CEP: $31.270-901$.

2 Departamento de Pediatria. Faculdade de Medicina. Universidade Federal de Minas Gerais. Belo Horizonte, MG, Brasil 3 Associação de Assistência à Criança Deficiente (AACD), Uberaba, MG, Brasil.

${ }^{4}$ Curso de Especialização em Terapia Ocupacional. Departamento de Terapia Ocupacional. Universidade Federal de Minas Gerais. Belo Horizonte, MG, Brasil.

5 Departamento de Fisioterapia. Escola de Educação Física, Fisioterapia e Terapia Ocupacional. Universidade Federal de Minas Gerais, Belo Horizonte, MG, Brasil

\section{Resumo}

Objetivos: avaliar impacto da interação nascimento pré-termo e nível socioeconômico (NSE) da família no desempenho funcional e na independência em tarefas da rotina diária, em crianças de três anos de idade.

Métodos: estudo transversal com 40 crianças, alocadas em quatro grupos, conforme variáveis de risco biológico e de risco social alto e baixo. A habilidade e a independência das crianças foram avaliadas pelo teste funcional padronizado PEDI, nas áreas: auto-cuidado, mobilidade e função social.

Resultados: foram revelados fatores de interação significativos nas habilidades funcionais de mobilidade e na independência em função social. Contrastes mostraram diferenças significativas entre crianças pré-termo e a termo, de NSE baixo. Crianças nascidas pré-termo apresentaram escores inferiores nos dois aspectos de desempenho funcional. Não foram observadas diferenças significativas nos grupos de crianças de NSE alto, nas áreas avaliadas.

Conclusões: o risco social (fator moderador) modificou a relação entre risco biológico e habilidades de mobilidade e independência em função social. A interação entre fatores biológicos e sociais, $e$ não apenas a presença de único fator de risco, influencia o desempenho funcional aos três anos de idade. Os resultados ampliam ações terapêuticas para crianças expostas a fatores de risco biológico e social.

Palavras-chave Prematuro, Desenvolvimento infantil, Fatores socioeconômicos, Fatores de risco, Fatores biológicos 


\section{Introdução}

Nos últimos anos, observou-se diminuição na taxa de mortalidade infantil atribuída aos avanços tecnológicos na área de neonatologia. ${ }^{1-3}$ Consequentemente, crianças clinicamente vulneráveis têm conseguido sobreviver e essa realidade pode contribuir para o aumento da morbidade nesse grupo de crianças, resultando em distúrbios ou atrasos no desenvolvimento. Enquanto o risco biológico define os principais fatores que influenciam os desfechos do desenvolvimento infantil em crianças de países desenvolvidos, no Brasil, a crescente morbidade observada em crianças que apresentaram intercorrências clínicas ao nascimento pode ser resultado da associação de fatores biológicos e sociais. Na verdade, dados epidemiológicos nacionais e internacionais revelam uma maior prevalência de nascimentos pré-termo em famílias de nível socioeconômico baixo.4,5

Existem diferentes fatores de risco para atraso no desenvolvimento da população infantil. De acordo com Benn, 6 crianças que se encontram em risco para atraso no desenvolvimento são aquelas expostas a fatores de risco biológico e/ou ambiental. Diversas evidências na literatura indicam que, dentre os fatores de risco biológico, a idade gestacional e o peso ao nascimento representam fatores preditivos importantes no prognóstico do desenvolvimento infantil. $3,5,7$

Além dos fatores de risco biológico, grande parte das crianças brasileiras que tiveram nascimento prétermo são de famílias nível socioeconômico baixo. Diversos fatores como características do ambiente físico (e.g. estímulos), escolaridade dos pais, dinâmica familiar, poder aquisitivo da família e relações familiares podem influenciar nos desfechos do desenvolvimento infantil.6,8-11 Em estudo realizado por Lima et al., 11 que avaliou crianças provenientes de classes socioeconômicas baixas de cidade do Nordeste brasileiro, observou-se que os fatores ambientais exercem importante influência no desenvolvimento motor e mental de crianças aos 12 meses de idade.

Apesar das influências de fatores de risco biológico e social estarem documentadas na literatura, alguns autores sugerem que seu impacto pode manifestar-se com intensidade variada, em diferentes etapas do desenvolvimento.3,6,12 Por exemplo, enquanto fatores biológicos como complicações perinatais e prematuridade são importantes nos desfechos do primeiro ano de vida, a influência do ambiente torna-se mais evidente após o segundo ano.3,6,12 Além do impacto diferenciado desses fatores de risco no contínuo do desenvolvimento, outros autores argu- mentam que esse impacto pode variar nas diferentes áreas do desenvolvimento infantil. ${ }^{10}$ Levy-Shiff $e t$ al. 10 investigaram o desenvolvimento de adolescentes nascidos a termo e pré-termo e observaram a interação de fatores biológicos e sociais em áreas como coordenação motora, cognição (QI) e comportamento. Entretanto, a memória visual foi influenciada, principalmente, pelo risco biológico, enquanto os aspectos cognitivos sofreram influência predominante do risco social.

Grande parte da literatura sobre o impacto de fatores de risco no desenvolvimento infantil investigou o tema, avaliando separadamente fatores de risco biológico e social. ${ }^{5}$ Recentemente, alguns autores vêm argumentando que a interação dos dois fatores de risco pode potencializar os efeitos no desenvolvimento infantil.8,13,14 $\mathrm{Na}$ verdade, o risco social pode ser entendido como uma variável moderadora, que interfere e modifica a relação entre risco biológico e desfechos do desenvolvimento infantil.2,8 Com base nessa argumentação, estudos que investigam individualmente os dois tipos de fatores de risco estariam simplificando um modelo, na realidade, muito mais complexo. Segundo Kochanek, 8 a literatura mostra a influência de múltiplos fatores no desenvolvimento infantil, sendo importante a investigação tanto dos fatores centrados na criança quanto aqueles relacionados ao ambiente. Juntos, fatores biológicos (e.g. centrados na criança) e sociais (e.g. centrados no ambiente) modificam e potencializam suas influências, trazendo conseqüências positivas e/ou negativas para o desenvolvimento infantil.13,14

O conceito de duplo risco é discutido na literatura internacional, sugerindo que a presença de diferentes tipos de fatores de risco pode modificar a manifestação independente de cada fator. 15 Diversos autores demonstraram que crianças nascidas pré-termo são, geralmente, mais vulneráveis aos efeitos de ambientes desfavoráveis se comparadas a crianças nascidas a termo. ${ }^{13,15}$ Alguns autores brasileiros começam a disponibilizar evidências sobre a associação de fatores de risco biológico e social no desempenho de crianças brasileiras. Magalhães et al., ${ }^{2}$ constataram a influência de fatores de risco biológico em crianças brasileiras provenientes de famílias de baixo nível socioeconômico. Esses autores verificaram que, na presença de duplo risco (social e biológico), crianças nascidas com idade gestacional entre 32 e 36 semanas (menor risco biológico) apresentaram dificuldades nas mesmas áreas do desenvolvimento em relação às nascidas com idades gestacionais inferiores a 32 semanas (maior risco biológico). Tais resultados ilustram os efeitos dos fatores de risco social no desenvolvimento de crianças expostas a diferentes intensi- 
dades ou gradientes de risco biológico.

O efeito de fatores de risco social na relação entre risco biológico e desenvolvimento infantil pode ser entendido como um efeito moderador. De acordo com Baron e Kenny, ${ }^{16}$ um fator moderador é definido como uma terceira variável que afeta a direção e a força da relação entre variável independente e variável dependente. Dessa forma, pode-se argumentar que o risco social, enquanto variável moderadora, modificaria a relação entre as variáveis risco biológico e desenvolvimento da criança.

É importante ressaltar que, embora grande parte da literatura tenha investigado o impacto de fatores de risco (biológico ou social) no diagnóstico geral e aspectos do desenvolvimento cognitivo, 3,5,7,10 não existem dados sobre o impacto desses fatores no desempenho da criança em atividades funcionais de vida diária. Tal informação torna-se relevante, uma vez que ilustra a manifestação dos componentes motores e cognitivos no cotidiano da criança e de sua família. Uma outra limitação importante a ser apontada é a predominância de evidências estrangeiras, dificultando a generalização dos resultados para crianças brasileiras. O risco social pode apresentar-se mais evidente no desenvolvimento de nossas crianças do que nas nascidas em países desenvolvidos.

O objetivo do presente estudo foi avaliar o efeito moderador do risco social na relação entre risco biológico e desempenho funcional. Especificamente, se avaliou o impacto da interação entre nascimento pré-termo e nível socioeconômico da família no desempenho de atividades funcionais e na independência em realizar tarefas da rotina diária, em crianças na faixa etária de três anos.

\section{Métodos}

\section{Participantes}

Para o presente estudo transversal, foram selecionadas 40 crianças com idade cronológica entre três anos e três anos e 11 meses. Essas crianças foram distribuídas em quatro grupos distintos, de acordo com a presença de fatores de risco biológico e social. Para investigação do impacto do risco biológico no desenvolvimento infantil, foram incluídas crianças que nasceram com idade gestacional inferior/igual a 32 semanas e peso ao nascimento igual ou inferior a 1500 gramas (grupo pré-termo) e crianças com idade gestacional superior a 37 semanas e peso ao nascimento superior a 2500 gramas (grupo a termo). A seleção das crianças nascidas pré-termo excluiu aquelas que apresentaram complicações peri- natais, tais como hemorragia intracraniana, hipóxia neonatal e outras condições que pudessem colocá-las em risco para distúrbios neuromotores. Para avaliação do impacto do risco social, as crianças que participaram desse do estudo foram estratificadas em dois grupos de acordo com a classe socioeconômica das famílias (alto e baixo), utilizando-se os critérios da Associação Brasileira de Institutos de Pesquisa de Mercado. ${ }^{17} \mathrm{O}$ nível socioeconômico foi avaliado por meio de um questionário estruturado, incluindo informações sobre a escolaridade dos pais, os bens de consumo da família (i.e., número de equipamentos eletrodomésticos, carro), presença empregada mensalista, entre outros. Cada item foi avaliado em uma escala 0 a 5 pontos, e a soma dessa pontuação forneceu um escore final. Esse escore final foi convertido em categorias que representam classes socioeconômicas distintas. As categorias socioeconômicas variam de A (muito alto) a $\mathrm{E}$ (muito baixo) e as demais categorias (B, C, D) indicam níveis socioeconômicos intermediários.

Foram excluídas deste estudo crianças que apresentaram diagnóstico clínico de retardo mental, deficiências visuais ou auditivas, distúrbios neurológicos ou padrões de movimento suspeitos ou anormais. As 40 crianças foram alocadas em quatro grupos: a) Grupo 1: crianças de alto risco biológico (nascimento pré-termo), cujas famílias pertenciam às classes socioeconômicas C e D (NSE baixo); b) Grupo 2: crianças de alto risco biológico, pertencentes a famílias de classes socioeconômicas A e B (NSE alto); c) Grupo 3: crianças de baixo risco biológico (nascimento a termo), cujas famílias pertenciam às classes socioeconômicas C e D (NSE baixo); d) Grupo 4: crianças de baixo risco biológico (nascimento a termo), pertencentes a famílias de classes socioeconômicas A e B (NSE alto).

\section{Instrumentação}

Todas as crianças que participaram desse estudo foram avaliadas pelo teste funcional infantil norteamericano PEDI (Pediatric Evaluation of Disability Inventory). ${ }^{18}$ Esse teste foi traduzido para o português e adaptado às especificidades culturais brasileiras, com a permissão e a colaboração dos autores estrangeiros. $19 \mathrm{O}$ PEDI é administrado no formato de entrevista estruturada com um dos cuidadores da criança. Ele informa sobre o perfil funcional de crianças entre seis meses e sete anos e seis meses de idade, em três níveis de função: auto-cuidado, mobilidade e função social. O perfil funcional documentado pelo PEDI avalia as habilidades disponíveis no repertório da criança para desempenhar atividades e 
tarefas de sua rotina diária (Parte I), bem como o seu nível de independência ou a quantidade de ajuda fornecida pelo cuidador (Parte II) e as modificações do ambiente utilizadas no desempenho funcional (Parte III).

A Parte I do teste avalia habilidades da criança em três escalas funcionais. A escala de auto-cuidado inclui 73 itens ou atividades funcionais, agrupadas nas seguintes tarefas: alimentação; higiene pessoal; banho; vestir/despir; uso do toilete; continência urinária e fecal. A escala de mobilidade inclui 59 itens ou atividades funcionais, agrupadas nas seguintes tarefas: transferências; locomoção dentro de um ambiente; locomoção em ambientes externos; uso de escadas. A escala de função social consiste de 65 itens agrupados nas seguintes tarefas: compreensão funcional; expressão funcional; resolução de problemas; brincar; auto-informação; orientação temporal; participação em tarefas domésticas; noções de auto-proteção; função na comunidade. Cada item, que constitui as escalas dessa parte, é avaliado com escore 1 (se a criança for capaz de desempenhar a atividade em sua rotina diária) ou escore 0 (se a criança não for capaz de desempenhá-la). Nessa primeira parte do teste PEDI, a pontuação dada aos itens que compõem cada escala é somada, resultando em um escore total bruto para cada área de função.

A Parte II do teste PEDI avalia a independência funcional da criança, que é uma medida inversa da quantidade de ajuda ou assistência fornecida pelo cuidador no desempenho de oito tarefas de autocuidado, sete tarefas de mobilidade e cinco tarefas de função social. A quantidade de assistência do cuidador é mensurada em escala ordinal que varia de 0 (indicando necessidade de assistência total) a cinco (a criança é independente no desempenho), com graduações intermediárias indicando níveis intermediários de ajuda (máxima, moderada, mínima e supervisão). Da mesma forma, como na Parte I, a pontuação dada para as tarefas em cada uma das três áreas funcionais é somada, resultando em três escores totais brutos de independência.

A Parte III do teste documenta a freqüência de modificações do ambiente utilizadas no desempenho das mesmas tarefas funcionais descritas acima (Parte II). Neste estudo, foram utilizadas somente as escalas das Partes I e II do teste. Estudos de validade e confiabilidade revelaram coeficientes elevados, indicando que esse é um teste válido e fidedigno. ${ }^{20,21}$

\section{Procedimento}

As crianças dos quatro grupos foram avaliadas por dois examinadores previamente treinados na administração do teste funcional PEDI. Após o treinamento, a consistência entre os examinadores foi testada, utilizando-se dez crianças com desenvolvimento normal e obtendo-se índices elevados de concordância (coeficientes de correlação intra-classe, ICC) foram superiores a 0,90 nas seis escalas do teste).

A avaliação das crianças foi realizada através de entrevista estruturada com os pais, entre agosto de 2002 e fevereiro de 2003. Antes da avaliação de cada criança, os pais ou responsáveis foram informados sobre os procedimentos, tiveram suas dúvidas esclarecidas e foram solicitados a assinar um termo de consentimento para participação do(a) filho(a) no estudo. Este estudo foi aprovado pelo Comitê de Ética em Pesquisa da Universidade Federal de Minas Gerais (UFMG), Brasil, em 24 de julho de 2002 e pelo Departamento de Ensino, Pesquisa e Extensão do Hospital das Clínicas (DEPE) da UFMG em 16 de agosto de 2002.

As crianças participantes foram selecionadas na região metropolitana de Belo Horizonte com base nos critérios citados anteriormente. Crianças dos Grupos 1 e 2 foram recrutadas no Ambulatório de Acompanhamento da Criança de Risco do Ambulatório Bias Fortes da UFMG e em consultórios médicos particulares (pediatras). As crianças dos Grupos 3 e 4 foram selecionadas em creches e escolas públicas e particulares da cidade de Belo Horizonte.

\section{Análise estatística}

Medidas de tendência central (média), de dispersão (desvio padrão) e freqüência foram usadas para descrever as características das crianças de cada grupo em relação às variáveis idade, sexo, peso ao nascimento, idade gestacional e nível socioeconômico da família.

De acordo com a literatura, o efeito de uma variável moderadora na relação entre duas outras variáveis é analisado por meio de fator de interação, como observado no modelo ANOVA fatorial. 16 Para avaliar os efeitos individuais dos fatores risco biológico (RB) e risco social (RS), bem como efeitos dos fatores de interação risco biológico $\mathrm{x}$ risco social (RB X RS), foram utilizadas análises de variância fatorial (Two-Way ANOVA) nas seis variáveis dependentes do estudo, que informam sobre habilidades e independência em cada uma das três áreas de função (auto-cuidado, mobilidade e função social). Para essas análises inferenciais, foi utilizado o pacote estatístico SUPERANOVA®. Quando o resultado do teste ANOVA revelou existência de 
diferença significativa, contrastes pré-planejados foram utilizados para localizar as diferenças entre duas médias. Em todas as análises foi considerado nível de significância $\alpha=0,05$.

\section{Resultados}

A Tabela 1 apresenta informação descritiva dos quatro grupos de crianças em relação às variáveis idade, peso ao nascimento, idade gestacional, sexo e classificação do nível socioeconômico das famílias.

Análise descritiva dos grupos de crianças pré-termo e a termo, de níveis socioeconômicos baixo e alto, nas variáveis idade, sexo, idade gestacional, peso ao nascimento e nível socioeconômico da família.

\begin{tabular}{|c|c|c|c|c|c|}
\hline \multirow[b]{3}{*}{ Variáveis } & & \multicolumn{4}{|c|}{ Grupos } \\
\hline & & \multicolumn{2}{|c|}{ Pré-termo } & \multicolumn{2}{|c|}{ Termo } \\
\hline & & NSE baixo & NSE alto & NSE baixo & NSE alto \\
\hline Idade*(meses) & & $39,7(4,47)$ & $41,8(3,58)$ & $42,0(2,30)$ & $42,2(2,94)$ \\
\hline \multirow[t]{2}{*}{ Sexo** } & Feminino & 9 & 5 & 5 & 6 \\
\hline & Masculino & 1 & 5 & 5 & 4 \\
\hline PN (gramas)* & & $1158(316)$ & $1274(263)$ & $>2500$ & $>2500$ \\
\hline IG (semanas)* & & $29,5(1,5)$ & $29,2(1,14)$ & $>36$ & $>36$ \\
\hline \multirow[t]{6}{*}{ NSE ** } & $\mathrm{A} 1$ & 0 & 2 & 0 & 2 \\
\hline & $\mathrm{A} 2$ & 0 & 5 & 0 & 3 \\
\hline & B1 & 0 & 2 & 0 & 3 \\
\hline & B2 & 0 & 1 & 0 & 2 \\
\hline & C & 8 & 0 & 4 & 0 \\
\hline & $\mathrm{D}$ & 2 & 0 & 6 & 0 \\
\hline
\end{tabular}

* Números indicam média (desvio padrão); * Números indicam freqüência de crianças em cada categoria; $\mathrm{NSE}=$ Nível sócio econômico (categorias: $\mathrm{A} 1=$ muito alto, $\mathrm{A} 2=$ alto, $\mathrm{B} 1$ = médio-alto, $\mathrm{B} 2=$ médio, $\mathrm{C}=$ médio-baixo, $\mathrm{D}=$ baixo); IG = idade gestacional ao nascimento; $\mathrm{PN}=$ peso ao nascimento.

Análises de variância fatorial (Two-Way ANOVA) foram utilizadas para examinar o efeito moderador do risco social (nível socioeconômico) na relação entre risco biológico (nascimento pré-termo) e desenvolvimento infantil (habilidades funcionais e independência) através dos efeitos de interação. Os resultados revelaram fatores de interação significativos em dois aspectos do desempenho funcional, especificamente, nas habilidades funcionais de mobilidade $(\mathrm{F}=4,567 ; p=0,0395)$ e na independência em função social $(\mathrm{F}=10,715 ; p=0,0024)$. Considerando-se o nível de significância utilizado nas análises inferenciais ( $\alpha=0,05$, teste não direcional), os resultados referentes aos fatores de interação desse estudo, que contou com uma amostra de $n=40$ (quatro grupos), apresentaram poder estatístico superior a
0,99.22 Ambos os efeitos significativos encontrados foram de grande magnitude (d superior a 0,80 ).

Nas habilidades de mobilidade, os contrastes pré-planejados revelaram diferenças significativas entre crianças dos Grupos 1 e 3, sendo que as crianças do Grupo 1 apresentaram desempenho inferior $(\mathrm{F}=48,56 ; p=0,0001)$. Não foram observadas diferenças significativas nos Grupos 2 e 4, nessa área de desempenho funcional ( $\mathrm{F}=1,65 ; p=0,207$ ). Entretanto, com relação à assistência do cuidador, observou-se diferença significativa entre grupos. As crianças do Grupo 2 apresentaram escores inferiores às do Grupo $4(\mathrm{~F}=5,56 ; \mathrm{p}=0,024)$. A mesma diferença foi observada em crianças dos Grupos 1 e 3 (F $=6,09 ; p=0,019)($ Figuras 1 e 2). 
Efeito moderador do risco social na relação entre risco biológico e desempenho funcional nas habilidades de mobilidade, em crianças de três anos.

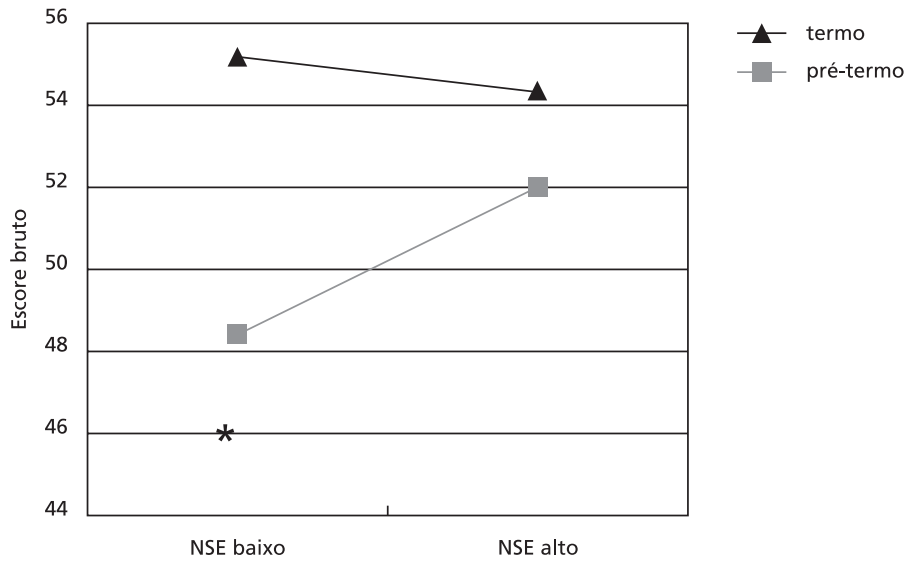

ANOVA. Interação: $\mathbf{F}=4,567 \quad(p=0,039)$

* ilustra diferença significativa entre grupos nos testes contrastes pré-planejados, $p<0,05$ ).

\section{Figura 2}

Efeito moderador do risco social na relação entre risco biológico e assistência do cuidador em mobilidade

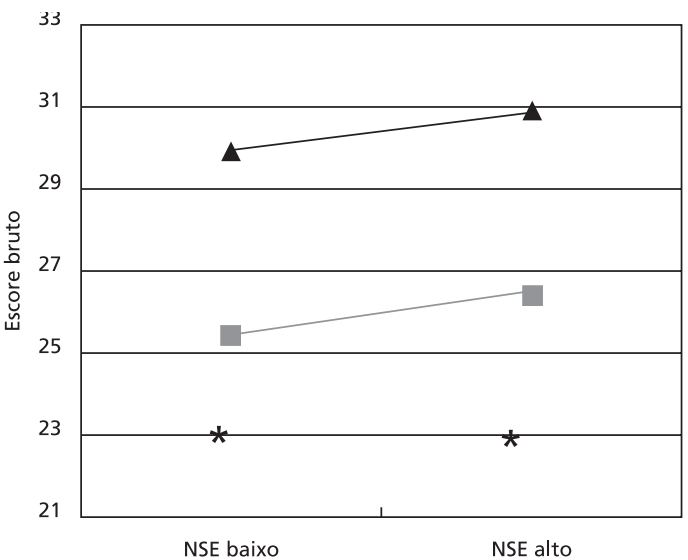

A termo

pré-termo

* Nesta figura, maior escore bruto indica menor assistência do cuidador (ou seja, maior independência da criança) no desempenho funcional na área de mobilidade em crianças de três anos; ANOVA. Interação: $\mathrm{F}=0,006(p=0,938)$;

* * ilustra diferença significativa entre grupos nos testes contrastes pré-planejados, $p<0,05$ ). 
Em relação às habilidades funcionais de função social, não foram evidenciadas diferenças significativas entre crianças a termo e pré-termo, dos diferentes níveis socioeconômicos. Entretanto, na independência de função social, foram observadas diferenças significativas na quantidade de assistência recebida pelas crianças do Grupo 1 em relação às crianças do Grupo 3. O Grupo 1 apresentou escores inferiores ao Grupo $3(\mathrm{~F}=14,20 ; \mathrm{p}=0,0006)$. Crianças dos Grupos 2 e 4 não apresentaram diferença significativa na quantidade de ajuda recebida do cuidador $(\mathrm{F}=0,74$; $\mathrm{p}=0,40)$ (Figura 3).

\section{Figura 3}

Gráfico ilustrativo do efeito moderador do risco social na relação entre risco biológico e assistência do cuidador em função social.

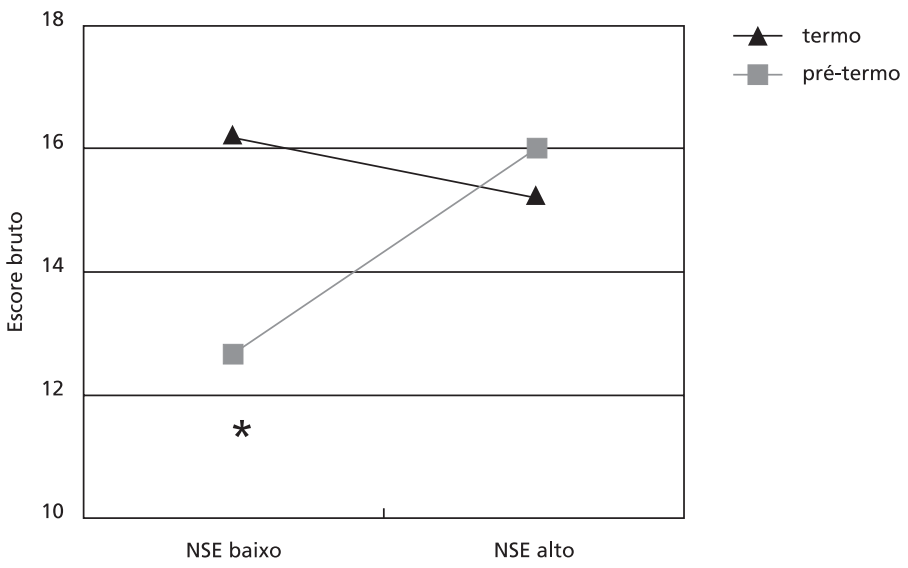

* Nesta figura, maior escore bruto indica menor assistência do cuidador (ou seja, maior independência da criança) no desempenho funcional na área de função social em crianças de três anos; ANOVA. Interação: $\mathrm{F}=10,715(p=0,002)$;

** ilustra diferença significativa entre grupos nos testes contrastes pré-planejados, $p<0,05)$.

Não foram observadas diferenças significativas nas habilidades funcionais de auto-cuidado entre grupos. Da mesma forma, não foram encontradas diferenças significativas em relação à quantidade de assistência oferecida pelo cuidador nessa área de função.

\section{Discussão}

Os resultados deste estudo revelam que o risco social, enquanto fator moderador, modificou a relação entre risco biológico e desenvolvimento infantil, em áreas específicas do desempenho funcional de crianças na faixa etária de três anos. A ação moderadora do risco social foi evidenciada nas habilidades de mobilidade e na independência em função social. Tais resultados ilustram a complexidade do desenvolvimento infantil, sugerindo que a análise de desfechos do desenvolvimento deve considerar essa complexidade na definição da proposta teóricometodológica de investigações científicas.

Em uma perspectiva transacional, o desenvolvimento infantil pode ser entendido como produto de interações dinâmicas entre a criança, a família e o contexto. ${ }^{23}$ Essa perspectiva amplia abordagem linear e unidirecional de modelos tradicionais, reconhecendo a influência da interação de diversos fatores nos desfechos infantis. Estudos que investigaram a relação entre família e desempenho da criança revelaram que os pais são agentes moduladores das experiências de seus filhos. A ação desses 
cuidadores é influenciada por fatores do contexto sociocultural da família. ${ }^{24,25}$ Assim, as interações sociais e o ambiente familiar no qual a criança está inserida podem incentivar ou limitar tanto a aquisição de habilidades quanto a independência funcional.

Neste estudo, a interação entre fatores de risco biológico e social foi observada em duas áreas de desempenho funcional: habilidades de mobilidade e independência em função social. Embora nessas duas áreas as crianças de baixo risco biológico nascidas a termo (Grupo 3) tenham demonstrado desempenho significativamente superior às crianças de alto risco biológico nascidas pré-termo (Grupo 1), essa diferença diminuiu consideravelmente quando os grupos eram provenientes de famílias de nível socioeconômico elevado (Grupos 2 e 4).

O elevado NSE das famílias está relacionado a determinadas condições favoráveis como maior escolaridade dos pais, maior acesso à informação e maior poder aquisitivo. Os resultados apresentados sugerem que tal contexto sociocultural poderia minimizar ou neutralizar eventuais prejuízos da performance motora, decorrentes da prematuridade, compensando os efeitos do risco biológico. Esse achado está de acordo com Schreiber et al.,26 que observaram que à medida que os recursos familiares aumentam, os pais tornam-se mais aptos a dar atenção e a investir em seus filhos, seguindo as recomendações de profissionais das áreas de saúde e educação. Assim, crianças de alto risco biológico e baixo risco social teriam maiores oportunidades de realizar atividades esportivas e de lazer, que favoreceriam a aquisição de habilidades motoras e, consequentemente, estimulariam desempenho semelhante ao de crianças de baixo risco biológico.

No que se refere à independência no desempenho de atividades funcionais na área de mobilidade, não se constatou o efeito moderador do risco social na relação entre risco biológico e independência. $\mathrm{Na}$ verdade, os resultados mostraram que a independência nas atividades de mobilidade de crianças nascidas pré-termo de NSE baixo é significativamente inferior à de crianças nascidas a termo, tanto em famílias de NSE alto quanto nas de NSE baixo. Dessa forma, apesar do repertório de habilidades de mobilidade ser semelhante nos dois grupos provenientes de famílias de nível socioeconômico alto (Grupos 2 e 4), crianças que nasceram pré-termo (Grupo 2) recebem mais ajuda para realização dessas tarefas do que as crianças nascidas a termo (Grupo 4). Caso o elevado NSE seja considerado um fator que contribui para a minimização do risco biológico nas habilidades de mobilidade, esse mesmo fator estaria limitando o desempenho independente, em crianças de alto risco biológico. Embora a argumentação apresentada acima, com base nos dados deste estudo, possa parecer contraditória, essas evidências ilustram a complexidade observada nas transações dos diversos fatores em diferentes aspectos do desenvolvimento infantil.

A literatura indica que, na relação entre a criança em risco para atraso ou distúrbio do desenvolvimento e seu cuidador, os últimos frequientemente tendem a exercer papel dominante nessa interação por um longo período, quando comparados a cuidadores de crianças que não são expostas a tais riscos. $27 \mathrm{~A}$ ajuda excessiva, fornecida pelos cuidadores de crianças pré-termo na execução de atividades funcionais, pode ocorrer por diferentes razões. Segundo Barnard e Kelly,27 esse comportamento dos pais pode ser atribuído à falta de resposta das crianças, ou seja, a maior participação dos pais nas atividades funcionais decorre da menor participação dos filhos. Outro fator que pode estar associado ao excesso de ajuda dos pais de crianças pré-termo nas atividades de mobilidade refere-se às expectativas dos mesmos quanto às necessidades de seus filhos, as quais podem se manifestar no sentido de subestimar as habilidades dessas crianças.

Um outro resultado importante deste estudo foi o efeito moderador do nível socioeconômico na relação entre risco biológico e independência em atividades de função social. Na presença de NSE baixo, crianças com nascimento pré-termo (Grupo 1) receberam maior assistência do cuidador para execução das tarefas funcionais, em relação às crianças a termo de mesmo NSE (Grupo 3), enquanto as com NSE alto receberam assistência semelhante, independente do nascimento ter sido a termo ou pré-termo. (Grupos 2 e 4). Tal resultado confirma as argumentações anteriores referentes às expectativas dos pais de diferentes níveis socioeconômicos acerca do desempenho independente da criança pré-termo. O baixo nível socioeconômico das famílias, geralmente associado a fatores como baixa escolaridade dos pais, pode contribuir para conclusões equivocadas sobre os efeitos da prematuridade. Dessa forma, a excessiva assistência dos pais de crianças pré-termo pode estar relacionada à dificuldade dos mesmos em compreender que a condição de vulnerabilidade biológica à qual essas crianças foram expostas no período neonatal não se perpetuam ao longo do desenvolvimento.

Estudos têm observado que as interações entre diversos fatores de risco e de proteção, e não a presença isolada de um determinado fator, influenciam de forma mais marcante o prognóstico do desenvolvimento infantil.23 $\mathrm{A}$ análise isolada de um fator 
de risco pode resultar em um modelo simplista que não reconhece a complexidade dos mecanismos envolvidos no desenvolvimento da criança. Investigações que avaliam o impacto de fatores de risco em desfechos do desenvolvimento infantil devem considerar, portanto, as diversas influências biológicas, sociais e ambientais envolvidas nesse processo. ${ }^{11,13}$ Assim, o desenvolvimento de habilidades e independência em diferentes áreas de função da criança pode ser considerado como resultado de uma complexa interação entre características intrínsecas da criança e influências externas provenientes dos ambientes físico e social.

A idade escolhida para o recorte transversal deste estudo (três anos) foi pautada na literatura. A primazia do risco biológico nos desfechos do desenvolvimento infantil, durante os dois primeiros anos de vida, é reconhecidamente aceita na literatura. 6,7 Com base nessa informação, é rotina nos serviços de acompanhamento infantil, a correção da idade gestacional de crianças que nascem prematuras, até a idade de 24 meses. A partir daí, os efeitos dos riscos biológicos não são preponderantes e, consequentemente, a emergência dos fatores de risco social começa a ser observada. Na idade escolar, os riscos sociais podem exercer influências negativas em alguns aspectos do desenvolvimento infantil, incluindo linguagem e rendimento escolar.6,10 Assim, optou-se por uma idade intermediária onde não houvesse expectativas de magnitudes diferenciadas nas influências dos fatores de risco biológico ou social.

Este estudo fornece informações importantes sobre o efeito moderador do risco social na relação entre risco biológico e desenvolvimento infantil. Entre- tanto, uma das limitações dessa investigação referese ao tipo de desenho metodológico. Por se tratar de um estudo transversal, é possível que a natureza e a dimensão das evidências encontradas possam ser alteradas ao longo do contínuo do desenvolvimento. Um estudo longitudinal forneceria informações mais precisas sobre o efeito moderador do nível socioeconômico das famílias, em diferentes faixas etárias do desenvolvimento de crianças expostas a fatores de risco biológico. Entretanto, enquanto estudo inicial, os resultados apresentados podem servir de ponto de partida para trabalhos subsequentes, que poderão adicionar informações e consolidar evidências.

O modelo transacional 23 reconhece a complexidade inerente ao desenvolvimento infantil. Além de sua aplicação norteando o desenvolvimento teóricometodológico de pesquisas científicas da área, esse modelo tem sido aplicado também na prática de diversos profissionais de saúde. Na verdade, o crescente interesse pelos fatores do contexto social e cultural, que se manifestam no desenvolvimento infantil, tem resultado em estudos acerca de avaliações e intervenções centradas na família, 11,28,29 e não somente na criança. Nessa perspectiva, as ações clínicas devem considerar tanto as informações intrínsecas da criança (fatores genéticos, complicações perinatais, baixo peso ao nascimento, prematuridade) quanto as características do ambiente das famílias (idade da mãe, NSE, educação dos pais.6,9 Os resultados apresentados neste estudo ressaltam a importância de identificar crianças expostas à múltiplos fatores de risco para ampliar ações de saúde direcionados a este grupo.

\section{Agradecimentos}

Ao Conselho Nacional de Desenvolvimento Científico e Tecnológico (CNPq).

\section{Referências}

1. Halpern LF, Brand, KL, Malone, AF. Parenting stress in mothers of very-low-birth-weight (VLBW) and full-term infants: a function of infant behavioral characteristics and child-rearing attitudes. J Pediatr Psychol 2001; 26:

93-104.

2. Magalhães LC, Barbosa VM, Paixão ML, Figueiredo EM, Gontijo APB. Acompanhamento ambulatorial do desenvolvimento de recém-nascidos de alto risco: característi- 
cas da população e incidência de seqüelas funcionais Rev Paul Pediatria 1998; 16: 191-6.

3. Miceli PJ, Goeke-Morey MC, Whitman TL, Kolberg KS, Miller-Loncar C, White RD. Brief report: birth status, medical complications, and social environment: individual differences in development of preterm, very low birth weight infants. J Pediatr Psychol 2000; 25: 353-8.

4. Barbieri MA, Silva AA, Bettiol LH, Gomes UA. Risk factors for the increasing trend in low birth weight among live births born by vaginal delivery. Rev Saúde Pública 2000; 34: 596-602.

5. Paixão ML, Mancini MC, Figueiredo EM, Ferreira APA, Gontijo, APB. O impacto da relação peso-idade gestacional no desenvolvimento do bebê pré-termo. Temas Desenvol 1994; 3: 54-60.

6. Benn R. Conceptualizing eligibility for early intervention In: Bryant DM, Graham MA. Implementing early intervention: from research to effective practice. New York: The Guilford; 1993. p. 18-45.

7. Pearl R, Donahue M. Brief report: four years after a preterm birth: children's development and their mothers' beliefs and expectations. J Pediatr Psychol 1995; 20: 363-70.

8. Kochanek TT. Enhancing screening procedures for infants and toddlers. In: Bryant DM, Graham MA. Implementing early intervention: from research to effective practice. New York: The Guilford; 1993. p. 46-66.

9. Koeppen-Schomerus G, Eley TC, Wolke D, Gringras P, Plomin R. The interaction of prematurity with genetic and environmental influences on cognitive developmen in twins. J Pediatr 2000; 137: 527-33.

10. Levy-Shiff R, Einat G, Mogilner MB, Lerman M, Krikler R. Biological and environmental correlates of developmental outcome of prematurely born infants in early adolescence. J Pediatr Psychol 1994; 19: 63-78.

11. Lima MC, Eickmann SH, Lima ACV, Guerra MQ, Lira PIC, Huttly SRA, Ashworth A. Determinants of mental and motor development at 12 months in a low income population: a cohort study in northeast Brazil. Acta Paediatr 2004. [in press].

12. Weiglas-Kuperus N, Baerts W, Smrkovsky M, Sauer PJ. Effects of biological and social factors on the cognitive development of very low birth weight children. Pediatrics 1993; 92: 658-65.

13. Grantham-McGregor SM, Lira PIC, Ashworth A, Morris SS, Assunção MA. The development of low birhtweight term infants and the effects of the environment in the North-East Brazil. J Pediatr 1998; 132: 661-6.

14. De Andraca I, Pino P, De la Parra A, Riveira F, Castillo M. Risk factors for psychomotor development among infants born under optimal biological conditions. Rev Saúde Pública 1998; 32: 138-47.

15. Hogan DP, Rogers ML, Msall ME. Functional limitations and key indicators of well-being in children with disability. Arch Pediatr Adolesc Med 2000; 154: 1042-8.
16. Baron RM, Kenny DA. The moderator-mediator variable distinction in social psychological research: conceptual, strategic and statistical considerations. J Pers Soc Psychol 1986; 51: 1173-82.

17. Associação Brasileira dos Institutos de Pesquisa e Mercado. Critério de classificação econômica Brasil: a volta de um critério único de classificação. Disponível em: http://www.aquanet.com.br/crit/ \%E9rio.htm. [2001 jun $11]$.

18. Haley SM, Coster WJ, Judlow LH, Haltiwanger JT, Andrellow PJ. Pediatric evaluation of disability inventory: development, standardization and administration manual. Boston: New England Medical Center; 1992.

19. Haley SM, Coster WJ, Judlow LH, Haltiwanger JT, Andrellow PJ. Inventário de avaliação pediátrica de disfunção: versão brasileira. Tradução e adaptação cultural: Mancini MC. Belo Horizonte: Laboratórios de Atividade e Desenvolvimento Infantil, Departamento de Terapia Ocupacional da Universidade Federal de Minas Gerais, 2000.

20. Feldman AB, Haley SM, Coryell J. Concurrent and construct validity of the pediatric evaluation of disability inventory. Phys Ther 1990; 70: 602-10.

21. Ganotti ME, Cruz C. Content and construct validity of a spanish translation of the pediatric evaluation of disability inventory for children living in Puerto Rico. Phys Occup Ther Pediatr 2001; 20: 7-24.

22. Cohen J. Statistical power analyses for the behavioral sciences. 2. ed. Hillsdale: Lawerence Erlbaum; 1998.

23. Sameroff AJ, Fiese BH. Transactional regulation and early intervention. In: Meisels SJ, Shonkoff JP. Handbook of early childhood intervention. New York: Cambridge University; 1990. p.119-49.

24. Kellegrew DH. Constructing daily routines: a qualitative examination of mothers with young children with disabilities. Am J Occup Ther 2000; 54: 252-9.

25. Brown SM, Humpry R, Taylor E. A model of the nature of family-therapist relationships: implications for education. Am J Occup Ther 1997; 51: 597-603.

26. Schreiber J, Effgen SK, Palisano RJ. Effectiveness of parental collaboration on compliance with a home program. Pediatr Phys Ther 1995; 7: 59-64.

27. Barnard KE, Kelly JF. Assessment of parent-child interaction. In: Meisels SJ, Shonkoff JP. Handbook of early childhood intervention. New York: Cambridge University; 1990. p. 278-302.

28. Darrah J, Law M, Pollock N. Family-centered functional therapy- a choice for children with motor disfunction. Inf Young Children 2001; 13: 79-87.

29. Eickmann SH, Lima ACV, Guerra MQ, Lima MC, Lira PIC, Huttly SRA, Ashworth, A. Improved cognitive and motor development in a community-based intervention of psychosocial stimulation in Northeast Brazil. Dev Med Child Neur 2003; 45: 536-41.

Recebido em 11 de novembro de 2003

Versão final apresentada em 14 de janeiro de 2004

Aprovado em 3 de fevereiro de 2004 